\title{
Multi-mode spatio-temporal instability in non-Boussinesq convection
}

\author{
Sergey A. Suslov*
}

(Received 8 August 2003, revised 15 March 2004)

\begin{abstract}
It is shown that nonlinear (non-Boussinesq) fluid property variations caused by large temperature differences between the walls of a vertical channel are responsible for the appearance of physically distinct types of instability in mixed convection flows: the previously known shear and new buoyancy-induced instabilities. Shear instability dominates the forced convection regimes, while the buoyancy instability prevails in nearly natural convection states. The most challenging situation requiring elaborate theoretical analysis and numerical verification arises in a mixed convection regime where both instabilities compete, forming a wide variety of possible flow patterns. Each of the instabilities is found to undergo the transition from a convective state (when disturbances grow and propagate away from their source)
\end{abstract}

*Dept. Maths \& Computing, University of Southern Queensland, Toowoomba, Australia. mailto: ssuslov@usq. edu.au

See http://anziamj.austms.org.au/V45/CTAC2003/Susl for this article, (c) Austral. Mathematical Soc. 2004. Published April 4, 2004; modified April 9, 2004. ISSN 1446-8735 
to absolute (when disturbances grow and occupy the complete flow domain). A number of regions in a complete parameter space corresponding to qualitatively different flow scenarios are identified, and accurate boundaries separating them are computed. Various patterns are illustrated by the direct evaluation of the Fourier integrals representing disturbances.

\section{Contents}

1 Introduction and problem definition

2 Outline of the theory of spatio-temporal instabilities and numerical approach

C153

3 Convective and absolute instabilities in high temperature convection

4 Conclusions

C161

References

C161

\section{Introduction and problem definition}

Mixed convection flows in a two-dimensional vertical channel have been the subject of intensive study for the last few decades, see works by Rudakov [5] and Fukui et al. [2] just to name a few. Normally the approach taken to analyze the resulting flow uses the Boussinesq approximation of the NavierStokes equations, which assumes no fluid properties variations except a linear density dependence on the temperature in the buoyancy term. The resulting solutions are relatively simple and can frequently be found analytically [4, 
e.g.]. But such an approach is valid only if the maximum temperature difference in the flow region does not exceed a few degrees Kelvin. This limitation is too restrictive for many practical applications such as chemical vapour deposition reactors. In this work the Low-Mach-Number (LMN) equations [1] are used to account for realistic fluid properties variations which lead to a rich variety of flow instability phenomena in mixed convection between two differentially heated vertical walls. The instabilities are caused by competing buoyancy and shear effects which were discussed in detail in $[6,7]$. In this paper we focus on the spatio-temporal nature of these instabilities.

Consider a flow of air with the average (reference) temperature $T_{r}=$ $300^{\circ} \mathrm{K}$ whose density $\rho$, dynamic viscosity $\mu$, thermal conductivity $k$ and specific heat $c_{p}$ nondimensionalised with respect to their values at $T_{r}$ vary with temperature $T$ according to the ideal gas and Sutherland formulas

$$
\rho T=1, \quad \mu=T^{3 / 2} \frac{1.368}{T+0.368}, \quad k=T^{3 / 2} \frac{1.648}{T+0.648}, \quad c_{p}=1,
$$

between two isothermal vertical plates separated by a gap of width $H$ and maintained at different temperatures $T_{h}$ and $T_{c}, T_{h}>T_{c}$. As discussed in [6] such flow is described by the LMN equations

$$
\begin{aligned}
& \frac{\partial \rho}{\partial t}+\frac{\partial \rho u_{i}}{\partial x_{i}}=0, \\
& \frac{\partial \rho u_{i}}{\partial t}+\frac{\partial \rho u_{i} u_{j}}{\partial x_{j}}=-\frac{\partial \Pi}{\partial x_{i}}+\frac{\mathrm{Gr}}{2 \epsilon}(\rho-1) n_{i}+\frac{\partial \tau_{i j}}{\partial x_{j}} \\
& \rho c_{p}\left(\frac{\partial T}{\partial t}+u_{j} \frac{\partial T}{\partial x_{j}}\right)=\frac{1}{\operatorname{Pr}} \frac{\partial}{\partial x_{j}}\left(k \frac{\partial T}{\partial x_{j}}\right),
\end{aligned}
$$

where the Grashof, Reynolds and Prandtl numbers and non-dimensional temperature difference between the walls are defined using the reference values:

$$
\begin{aligned}
& \mathrm{Gr}=\frac{\rho_{r}^{2} g\left(T_{h}-T_{c}\right) H^{3}}{\mu_{r}^{2} T_{r}}, \quad \operatorname{Re}=-\frac{\rho_{r} H^{3}}{12 \mu_{r}^{2}} \frac{d \Pi}{d y}, \\
& \operatorname{Pr}=\frac{\mu_{r} c_{p r}}{k_{r}}=0.71, \quad \epsilon=\frac{T_{h}-T_{c}}{2 T_{r}} .
\end{aligned}
$$



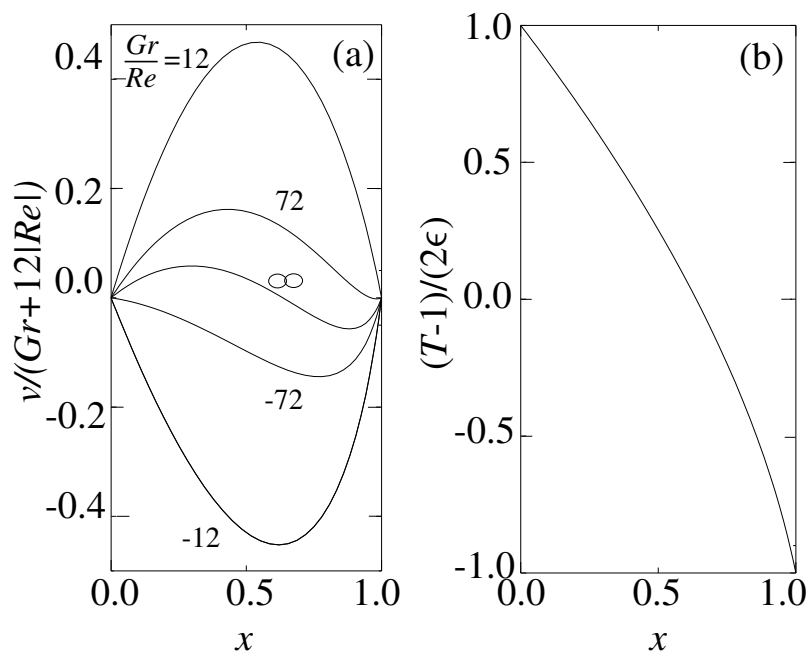

Figure 1: Basic flow velocity (a) and temperature (b) profiles for nonBoussinesq convection.

We investigate in detail the flow arising in the non-Boussinesq mixed convection regime at $\epsilon=0.6$. The steady $y$-independent parallel basic flow profiles are shown in Figure 1. The temperature difference between the walls in this regime is $360^{\circ} \mathrm{K}$ and the fluid transport properties vary significantly across the channel (up to 30\%), so that the temperature deviates significantly from a classical Boussinesq linear profile. In turn the nonlinear viscosity variation with temperature breaks the symmetry of velocity profiles, shifting them towards the cold wall. In addition, $v(x, \mathrm{Re}, \mathrm{Gr}) \neq v(-x,-\mathrm{Re}, \mathrm{Gr})$, that is, changing the direction of the applied pressure gradient leads to nontrivial changes in the basic flow velocity fields. For this reason the spatio-temporal instability results will be presented for both positive and negative values of the Reynolds number. 


\section{Outline of the theory of spatio-temporal instabilities and numerical approach}

Equations (1)-(4) are linearised about the basic flow solutions and discretized using the Chebyshev pseudo-spectral collocation method as described in [6]. The Fourier transform in the $y$-direction and the Laplace transform in time result in a matrix problem

$$
[\mathrm{A}(\alpha, x ; \operatorname{Re}, \mathrm{Gr}, \epsilon, \operatorname{Pr})-\sigma \mathrm{B}(x ; \epsilon)] \tilde{\mathbf{w}}(\alpha, x ; \operatorname{Re}, \operatorname{Gr}, \epsilon, \operatorname{Pr})=\mathbf{F}(x),
$$

where $\alpha$ is the longitudinal wavenumber, $\sigma$ is the complex disturbance amplification rate, $\tilde{\mathbf{w}}$ is the disturbance eigenfunction and $\mathbf{F}$ is the external forcing. For $\mathbf{F}=0$ equation (5) becomes a generalised singular matrix eigenvalue problem which represents an implicit numerical dispersion relation (NDR) $\sigma=\sigma(\alpha)$. The parametric surface $\Re(\sigma)=0$ defines the boundary of linear instability. For parameter values for which there exists a range of wavenumbers so that $\Re(\sigma(\alpha))>0$, we investigate the spatio-temporal character of instability by choosing localised $\mathbf{F} \neq 0$. The theoretical background of such a study is too complicated to be given here in detail and can be found elsewhere [3, e.g.]. In brief, upon applying the inverse Laplace and Fourier transforms to (5) and using the residue theorem we obtain a wave envelope response in the form

$$
\mathbf{w}(x, y, t)=\frac{1}{2 \pi} \int_{-\infty}^{\infty}\left\langle\mathbf{w}^{\dagger}(\alpha, x), \mathbf{F}(x)\right\rangle \tilde{\mathbf{w}}(\alpha, x) e^{\sigma(\alpha) t+i \alpha y} d \alpha,
$$

where $\mathbf{w}^{\dagger}$ is the adjoint eigenfunction defined with respect to some appropriately defined inner product $\langle$,$\rangle and the integration is performed along the$ real $\alpha$ line. The asymptotic behaviour of (6) is analysed using the method of steepest descent [3] by deforming the original $\alpha$ integration contour into the steepest descent path through the saddle point $\alpha_{s}$ of NDR defined by

$$
\left.\frac{d}{d \alpha}\left[\sigma_{j}(\alpha)\right]\right|_{\alpha=\alpha_{s}}=0
$$


in the complex $\alpha$ plane. The asymptotic value of (6) then is

$$
\mathbf{w}(x, y, t) \sim \frac{1}{\sqrt{t}}\left\{\frac{\left\langle\mathbf{w}^{\dagger}\left(\alpha_{s}, x\right), \mathbf{F}(x)\right\rangle}{\sqrt{\left|d^{2} \sigma / d \alpha^{2}\right|_{\alpha=\alpha_{s}}}} \tilde{\mathbf{w}}\left(\alpha_{s}, x\right) e^{\sigma\left(\alpha_{s}\right) t}\right\} .
$$

It defines the dynamics at a fixed location $y=0$, where the localised disturbance is introduced at $t=0$. Consequently, the spatio-temporal character of linearly unstable disturbances is determined by the sign of $\sigma^{R}\left(\alpha_{s}\right)$ : $\sigma^{R}\left(\alpha_{s}\right)>0(<0)$ corresponds to absolute (convective) instability and the task of determining the boundary between convective and absolute instabilities is mainly reduced to an iterative search of the saddle points satisfying (7) so that $\sigma^{R}\left(\alpha_{s}\right)=0$ (see [3] for other important details). It also can be shown that the magnitude of disturbance group speed $c_{g}=-d \Im(\sigma) / d \alpha$ is closely related to the character of the instability: systems with large group speed (relative to local basic flow velocity) are normally convectively unstable (see Figure 2) while wave envelopes characterised by relatively small group speed are likely to be absolutely unstable (see Figure 3). Finally, note that although the determination of the spatio-temporal character of instability seems to be similar to a well understood linear stability analysis, it is much more demanding and is a greatly more sophisticated numerical task whose full detail will be presented in a separate full length publication currently being prepared by the author.

\section{Convective and absolute instabilities in high temperature convection}

As discovered in [6], when the temperature difference between the walls is very large so that the fluid property variations become essentially nonlinear, two instability modes appear in the flow. The first mode (shear) is associated with the large velocity gradient near the inflection point of the 
velocity profile (for natural convection) or with Tollmien-Schlichting waves in the boundary layers (for forced convection). The direction and speed of its propagation are dictated by the direction and magnitude of the applied pressure gradient (Reynolds number). The second mode (buoyancy) arises because of the highly nonlinear variation of the basic flow temperature profile near the cold wall as seen in Figure 1(b). For small values of the Reynolds number it manifests itself in the form of lumps of overcooled heavier fluid sliding down along the cold wall. Because of this the buoyancy-driven instability has a preferred downward propagation direction, regardless of the sign of the Reynolds number when its magnitude is small. The typical dynamics of the buoyancy disturbance pattern is shown in Figure 2. It is obtained via the direct numerical integration of equation (6). This situation corresponds to a convectively unstable regime as the disturbance wave envelope (located between the two horizontal lines which bound the domain within which the thermal disturbance magnitude is above a specified fixed level) propagates away from its original location. It is only at Re $\gtrsim 594$ that the upward basic flow becomes strong enough to carry the buoyancy instability cells upward (the group speed becomes positive, see Figure 4(b). The buoyancy mode group speed is zero at $(\mathrm{Re}, \mathrm{Gr}) \approx(594,74391)$ so that the disturbance envelope expands without drifting. This scenario corresponds to the transition to absolute instability.

The group speed becomes zero at $(\operatorname{Re}, \mathrm{Gr}) \approx(22,15791)$ for the shear mode and this is the only set of parameters for which the shear mode becomes absolutely unstable as soon as it appears. A typical disturbance pattern for absolutely unstable shear mode is shown in Figure 3 (the edges of the disturbance envelope propagate in opposite directions). For values of the Reynolds number other than 22, a finite range of Grashof numbers exists where the flow is convectively unstable due to the shear induced disturbances.

In general, increasing the Reynolds number (pressure gradient) leads to a unidirectional basic flow (see Figure 1a) and increases its tendency to become 

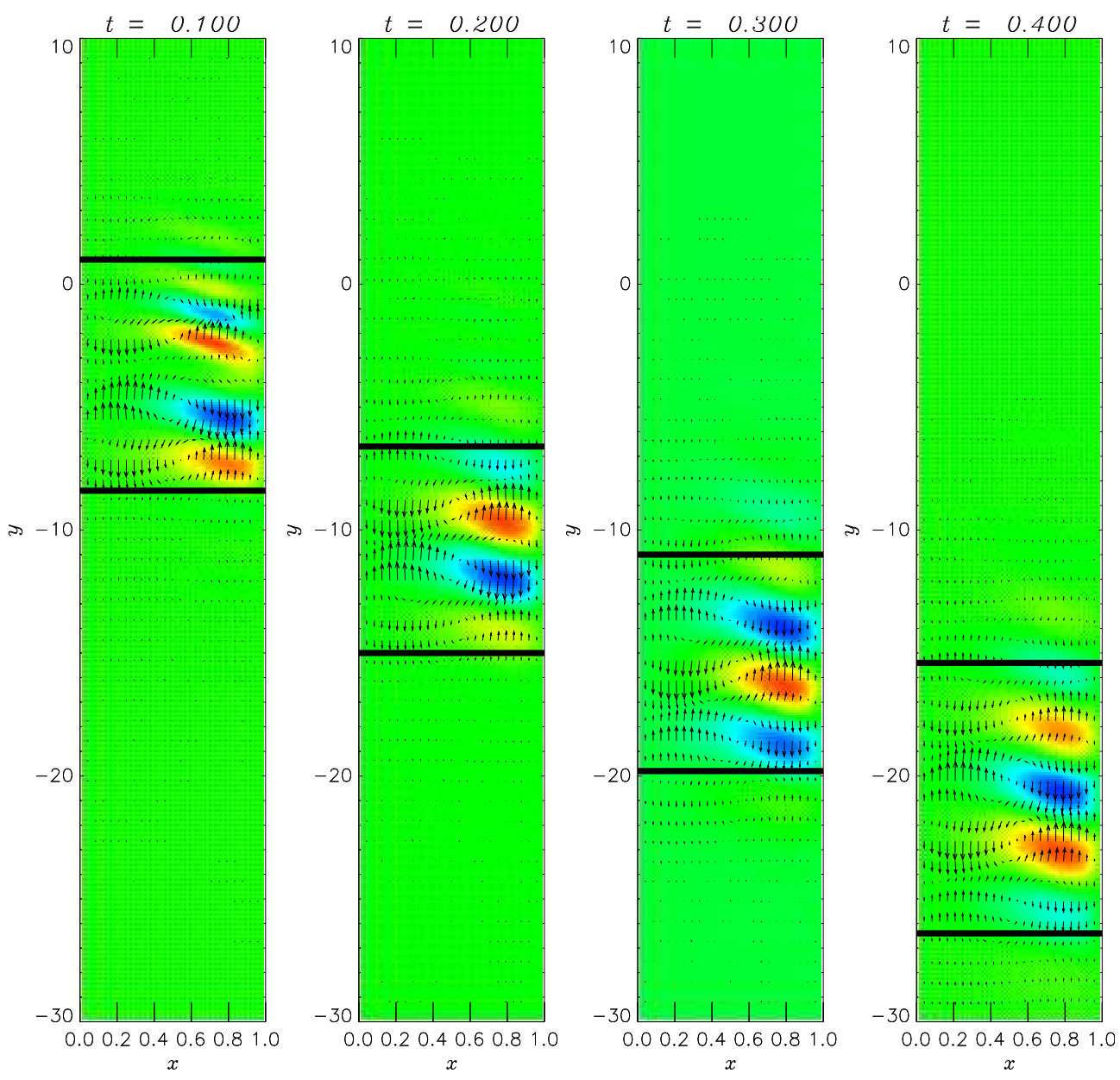

FigURE 2: (a) Evolution of convectively unstable buoyancy instability mode at $(\operatorname{Re}, \mathrm{Gr}, \epsilon)=(0,10000,0.6)$. Colours represent the disturbance thermal field. See the mpeg movie in http://anziamj.austms.org.au/V45/CTAC2003/Susl/ G10000r0e6.mpg 

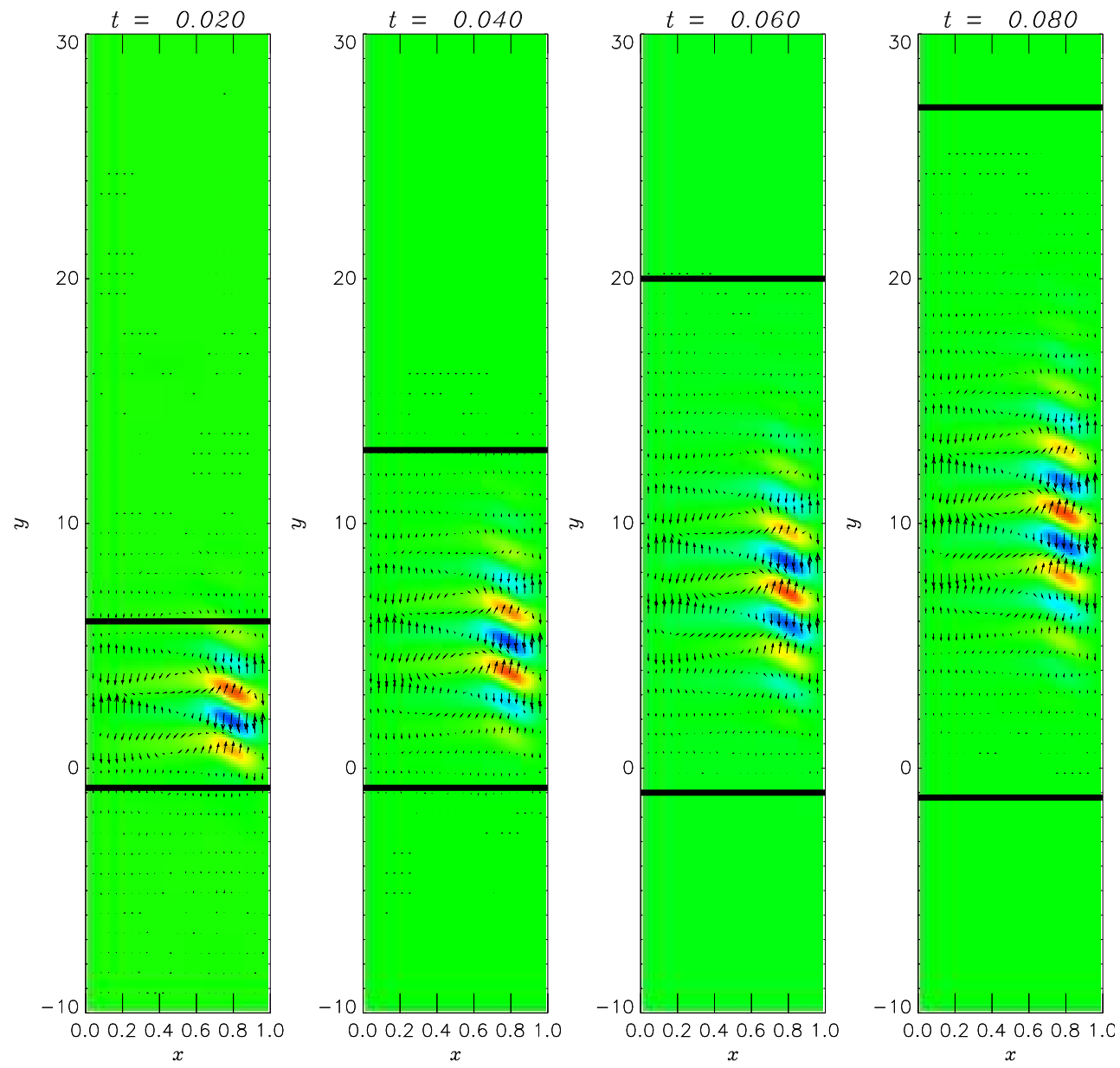

FiguRE 3: (a) Evolution of absolutely unstable shear instability mode at $(\operatorname{Re}, \mathrm{Gr}, \epsilon)=(260,50000,0.6)$. Colours represent the disturbance thermal field. See the mpeg movie in http://anziamj.austms.org.au/V45/CTAC2003/Susl/ G50000r260e6.mpg 

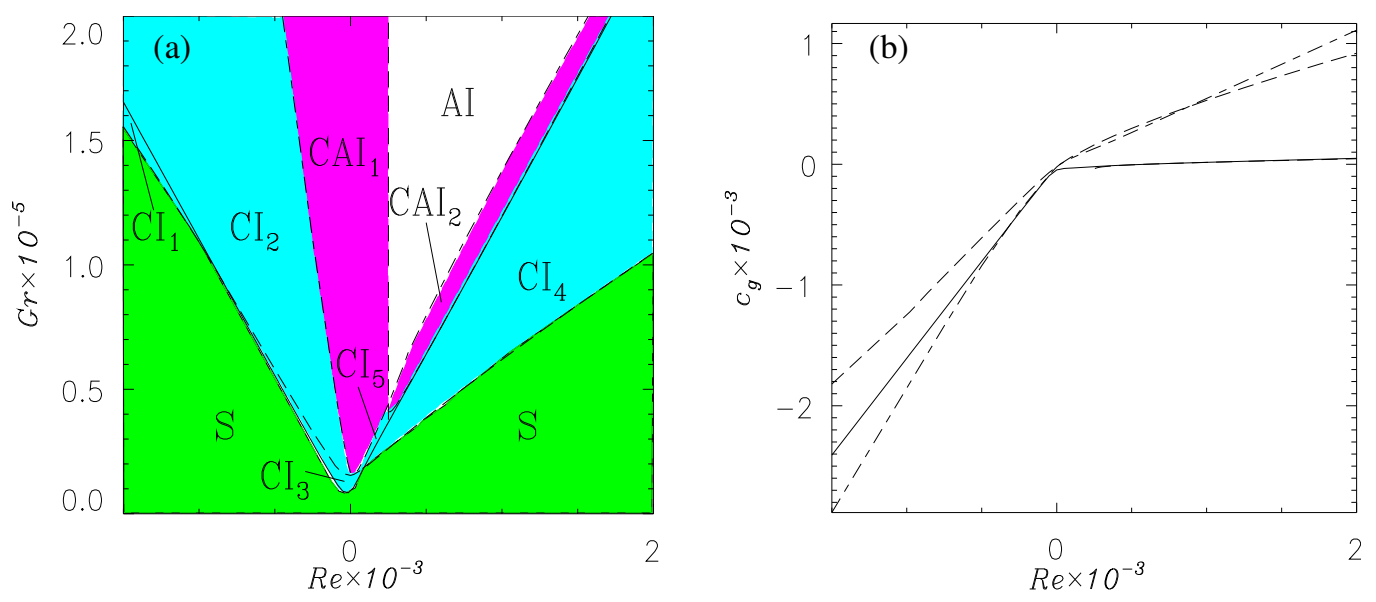

FIGURE 4: (a) Linear and absolute instability boundaries; (b) disturbance group speeds along the instability boundaries for non-Boussinesq mixed convection at $\epsilon=0.6$.

convectively unstable. In contrast, increasing the Grashof number leads to the basic flow reversal which can support disturbance propagation in both directions and thus encourages absolute instability. The transition between these two regimes in mixed convection is determined by a nontrivial balance between the competing effects of buoyancy and the pressure gradient. The presence of two physically distinct instability modes makes the combined stability diagram in the governing parameter space very complicated. It is summarised in Figure 4(a). All numerical values used in this diagram were determined using a spectral Chebyshev collocation approximation which guarantees high accuracy of the results so that the qualitative structure of all depicted regions is not affected by numerical errors. The individually labelled regions are discussed in detail below.

S. Linearly stable basic flow. It has been found in [7] that non-Boussinesq fluid property variations lead to subcritical bifurcations for a wide range of the Reynolds numbers, partially including that of Figure 4. This means that finite amplitude initial disturbances can trigger instability 
in some sub-domains of region $\mathbf{S}$, but in the context of the current investigation, we focus on infinitesimal disturbances for which the linear theory of convective and absolute instabilities is valid.

$\mathbf{C I}_{\mathbf{1}}$. This is a region of large negative Reynolds numbers where only the shear mode leads to linear convective instability while the buoyancy mode is linearly stable. Forced by the imposed longitudinal pressure gradient, the shear disturbances propagate downward (negative group speed, see Figure 4(b).

$\mathbf{C I}_{2}$. At the larger Grashof numbers the flow becomes linearly unstable with respect to both shear and buoyancy disturbances. They both propagate downward although with substantially different group speeds (see solid and dash-triple-dotted lines in the left part of Figure 4(b) and lead to convective instability as their spatial extension rate is not sufficiently large for disturbances to grow upstream.

$\mathbf{C I}_{3}$. In this region the imposed pressure gradient is weak so that the associated Reynolds numbers are relatively small. The flow is dominated by natural rather than forced convection. As a result the instability is driven only by the buoyancy force and arises near the cold wall, see [7]. Hence the disturbances propagate downward with relatively large group speed while their temporal amplification rate $\sigma^{R}$ remains small, as the region is close to the linear transition shown by the solid line. The combination of these conditions leads to convective instability.

$\mathbf{C I}_{\mathbf{4}}$. This relatively large region is similar to $\mathbf{C I}_{\mathbf{1}}$ except that the shear disturbances are forced to propagate upward by the imposed pressure gradient.

$\mathbf{C I}_{5}$. Here both shear and buoyancy modes are linearly and convectively unstable, but physically distinct disturbances propagate in the opposite directions: buoyancy - downward, shear - upward. This is explained by examining the basic flow velocity profile shown by the solid line in Figure 1(a). The buoyancy disturbances arising at the region of 
the large temperature gradient near the cold wall (see Figure 1b) are carried downwards by a primary flow while the shear disturbances arising in the region of large velocity gradient closer to the centreline of a channel are carried upwards. The degree of flow reversal and the disturbance amplification rates are not sufficiently large to enable an extension of the disturbance wave envelopes in the directions opposite to their propagation. Thus the instability remains convective for both envelopes. This means that the initially localised disturbance will be felt upstream as the shear component, and downstream as the buoyancy component, but it will eventually decay at any fixed spatial location.

$\mathbf{C A I}_{1}$. In this region above the dashed line and to the left of the vertical dotted line at $\mathrm{Re} \approx 255$, the convectively unstable buoyancy disturbances propagate downward. The temporal amplification rate for the shear disturbance here is relatively large so that the disturbance extension rate becomes sufficient to overcome the drift of the disturbance envelope with a positive group speed. Shear mode instability is absolute here.

$\mathbf{C A I}_{2}$. The situation here is similar to that in region $\mathbf{C A I}_{\mathbf{1}}$ except that the shear mode remains convectively unstable, as the Grashof number is not sufficiently large to force the strong flow reversal required for the absolute instability of the shear mode, see the dash-dotted line in Figure 1(a). Instead, the buoyancy mode becomes absolutely unstable as its group speed remains small. Disturbance envelopes of both types propagate upward.

AI. In this region above the dashed line and to the right of the vertical dotted line in Figures 4(a), both instability modes are absolutely unstable as the Grashof number is large enough to support a strong basic flow reversal, see the dashed line in Figure 1(a).

The absolute instability for the buoyancy mode for Re $\lesssim 255$ does not exist because in these regimes the strength of the applied pressure gradient is not sufficient to overcome the downward motion of overcooled fluid lumps. 


\section{Conclusions}

The nonlinear fluid property variations associated with large temperature differences in the flow region are shown to lead to a remarkably rich variety of spatio-temporal instabilities observed even in very simple geometries. The analysis of these instabilities requires careful analytical and elaborate numerical investigations whose full detail will be reported by the author elsewhere. The problem discussed offers significant theoretical interest which is enhanced by its direct relevance to industrial applications such as chemical vapour deposition reactors and thermal insulation systems.

\section{References}

[1] D. R. Chenoweth and S. Paolucci, Gas flow in vertical slots with large horizontal temperature differences, Phys. Fluids, 28, 2365-2374, 1985. C151

[2] K. Fukui, M. Nakajima, H. Ueda and T. Mizushina, Flow instability and transport phenomena in combined free and forced convection between vertical parallel plates, J. Chem. Engng Japan, 15, 172-180, 1982. C150

[3] P. Huerre and P. A. Monkewitz, Local and global instabilities in spatially developing flows, Ann. Rev. Fluid Mech., 22, 473-537, 1990. C153, C154

[4] G. D. McBain, Fully developed laminar buoyant flow in vertical cavities and ducts of bounded section, J. Fluid Mech., 401, 365-377, 1999. C151 
[5] R. N. Rudakov, Spectrum of perturbations and stability of convective motion between vertical plates, Appl. Math. Mech., 31, 376-383, 1967. C150

[6] S. A. Suslov and S. Paolucci, Stability of mixed-convection flow in a tall vertical channel under non-Boussinesq conditions, J. Fluid Mech., 302, 91-115, 1995. C151, C153, C154

[7] S. A. Suslov and S. Paolucci, Nonlinear stability of mixed convection flow under non-Boussinesq conditions. Part 1. Analysis and bifurcations, J. Fluid Mech., 398, 61-85, 1997. C151, C158, C159 